\title{
As contribuições da neurociência para a prática docente
}

\author{
The contributions of neuroscience to teaching practice \\ Las contribuciones de la neurociencia a la práctica docente
}

Recebido: 27/07/2021 | Revisado: 03/08/2021 | Aceito: 25/09/2021 | Publicado: 01/10/2021

\author{
Vitorina Gomes do Lago ${ }^{1}$ \\ ORCID: https://orcid.org/0000-0002-5036-422X \\ Universidade Municipal de São Caetano do Sul, Brasil \\ E-mail: vitoryna@hotmail.com \\ Diomark Pereira de Araujo ${ }^{2}$ \\ ORCID: https://orcid.org/0000-0003-0268-0794 \\ Universidade Federal do Oeste do Pará, Brasil \\ E-mail: diomark.araujo@ hotmail.com \\ Gilma da Silva Pereira Rocha ${ }^{3}$ \\ ORCID: https://orcid.org/0000-0002-2323-3902 \\ Universidade Luterana do Brasil, Brasil \\ E-mail: rochagsp@gmail.com \\ Maria Rodrigues Oliveira ${ }^{4}$ \\ ORCID: https://orcid.org/0000-0001-6656-1765 \\ Universidade Luterana do Brasil, Brasil \\ E-mail: ensino.semed2013@gmail.com
}

\begin{abstract}
Resumo
Este artigo teve objetivo demonstrar as contribuições da neurociência para a prática docente, bem como evidenciar como esse campo de estudo vem colaborando para um melhor entendimento de como se processa a aprendizagem no cérebro humano, permitindo aos professores repensarem a sua ação didática. Pensar em uma aprendizagem diferenciada, requer pensar em práticas inovadoras, espaços adequados e ambientes estimuladores, e de posse das informações apreendidas por essa nova área do conhecimento e indicações de conteúdos e técnica de trabalho, o professor poderá desenvolver seu planejamento de forma a possibilitar ao aluno uma aprendizagem significativa. Diante disso, foi utilizada uma metodologia qualitativa, por meio de análise de artigos, para descrever como a neurociência vem contribuindo para a prática docente no ambiente escolar. Dessa forma, os resultados demonstraram que a neurociência aliada a técnicas, estratégias de trabalho e um ambiente que estimulem o desejo pela busca de conhecimento, pode contribuir com o fortalecimento de uma aprendizagem significativa.
\end{abstract}

Palavras-chave: Neurociências; Prática docente; Educação; Aprendizagem; Ensino.

\begin{abstract}
This article aimed to demonstrate the contributions of neuroscience to teaching practice, as well as to show how this field of study has been contributing to a better understanding of how learning is processed in the human brain, allowing teachers to rethink their didactic action. Thinking about differentiated learning requires thinking about innovative practices, adequate spaces and stimulating environments, and having the information learned by this new area of knowledge and indications of content and work technique, the teacher will be able to develop their planning in order to enable the student a meaningful learning. Therefore, a qualitative methodology was used, through the analysis of articles, to describe how neuroscience has been contributing to teaching practice in the school environment. Thus, the results showed that neuroscience, combined with techniques, work strategies and an environment that encourages the desire to seek knowledge, can contribute to the strengthening of meaningful learning.
\end{abstract}

Keywords: Neurosciences; Teaching practice; Education; Learning; Teaching.

\footnotetext{
${ }^{1}$ Especialista em pedagogia Escolar: Administração, Orientação e Supervisão e professora da Rede Municipal de Itaituba, com formação em pedagogia.

${ }^{2}$ Mestre em Educação pela Universidade Federal do Oeste do Pará-UFOPA (2020). Graduado em Pedagogia pela Universidade Estadual Vale do Acaraú (2007), com habilitação em Orientação, Supervisão e Direção Escolar. Atualmente está como Diretor Escolar e professor da disciplina Ensino da Arte n município de Itaituba. E-mail: diomark.araujo@hotmail.com

${ }^{3}$ Doutoranda em Educação pela Universidade Luterana do Brasil- Campus Canoas. Mestra em Educação pela Universidade Federal do Pará. Especialista em Educação Especial e Inclusiva. Licenciada em Letras e Pedagogia. Professora da Educação Especial na SEMED- Santarém. Professora na Escola de Ensino Técnico do Estado do Pará. E-mail: rochagsp@gmail.com

${ }^{4}$ Mestranda pela Universidade Luterana do Brasil-ULBRA- Campus Canoas. Pedagoga Pela Universidade Federal do Pará-UFPA. Professora da Rede Municipal de Itaituba, Técnica em Assuntos Educacionais do Instituto Federal do Pará-IFPA e Tesoureira do Sindicato dos Trabalhadores de Itaituba-SINTEPP. Pertence ao Grupo de Pesquisa Cultura e Educação. E-mail: ensino.semed2013@gmail.com
} 


\begin{abstract}
Resumen
Este artículo tuvo como objetivo demostrar los aportes de la neurociencia a la práctica docente, así como mostrar cómo este campo de estudio ha venido contribuyendo a una mejor comprensión de cómo se procesa el aprendizaje en el cerebro humano, permitiendo a los docentes repensar su acción didáctica. Pensar en aprendizajes diferenciados requiere pensar en prácticas innovadoras, espacios adecuados y ambientes estimulantes, y teniendo la información aprendida por esta nueva área de conocimiento e indicaciones de contenido y técnica de trabajo, el docente podrá desarrollar su planificación con el fin de posibilitar el alumno un aprendizaje significativo. Por tanto, se utilizó una metodología cualitativa, a través del análisis de artículos, para describir cómo la neurociencia ha venido contribuyendo a la práctica docente en el ámbito escolar. Así, los resultados mostraron que la neurociencia, combinada con técnicas, estrategias de trabajo y un entorno que fomente el deseo de buscar conocimiento, puede contribuir al fortalecimiento de aprendizajes significativos.
\end{abstract}

Palabras clave: Neurociencias; Práctica docente; Educación; Aprendiendo; Enseñanza.

\title{
1. Introdução
}

A neurociência é um ramo do conhecimento que nos últimos anos vem se tornando objeto de estudos por partes das mais variadas áreas do conhecimento, em especial da educação. No campo educacional essa nova forma de pensar a aprendizagem e de como o conhecimento se processa no cérebro humano, tem contribuído com a prática docente no cotidiano escolar. Sendo assim, este artigo tem como objetivo demonstrar a importância da neurociência para a prática docente, destacando sua positividade para a aprendizagem do aluno.

Quando é discutido o procedimento educativo, é preciso colocar em foco os processos neurais, as redes que se estabelecem, neurônios que estão interligados e como são construídas as novas sinapses. Sendo assim, entende-se que neurociência é o estudo de como é realizado a aprendizagem no cérebro. É o caminho para se compreender como as redes neurais são processadas e chegam ao cérebro.

Para uma melhor compreensão, utilizou-se uma metodologia qualitativa a partir da técnica de leitura e análise de artigos, o que contribuiu para um melhor entendimento de como a neurociência tem contribuído com a prática docente e a aprendizagem do aluno. Diante disso, a pesquisa é de grande importância porque pode contribuir com aqueles que buscam compreender como a neurociência pode contribuir para a realização da prática docente no ambiente escolar.

\section{Metodologia}

A pesquisa é de natureza qualitativa e bibliográfica. De acordo com Godoy (1995), a pesquisa qualitativa ocupado um amplo reconhecimento entre as mais variadas possibilidades de estudar os fenômenos que envolvem o ser humano e suas intricadas relações vivenciadas em diversos ambientes. Para o alcance dos objetivos propostos, realizou-se um levantamento bibliográfico de livros, artigos científicos e sites relacionados ao tema, para descrever teorias que abordassem sobre a As Contribuições da Neurociência para a Prática Docente, mediante uma análise sistemática, com fichamento de cada obra ressaltando os pontos relevantes ao assunto. A pesquisa bibliográfica é desenvolvida com base em material já elaborado, construído, principalmente, por meio de livros e artigos científicos. Embora em quase todos os estudos seja exigido algum tipo de trabalho dessa natureza, há pesquisas desenvolvidas exclusivamente a partir de fontes bibliográficas (Gil, 2002, p.44). Em todo o processo de análise obteve-se resultados satisfatórios quanto aos dados coletados sobre as Contribuições da Neurociência para a Prática Docente, de modo a contribuir para um melhor entendimento de como o discente desenvolve sua aprendizagem no cotidiano escolar.

\section{Neurociência}

A neurociência surgiu por volta do século XIX, a partir dos estudos dos cientistas Santiago Ramon e Cajal, que descobriram a existência de neurônios que, de posse desse conhecimento, desenvolveram a teoria neural. Essa nova área do 
conhecimento, trata-se de uma ciência que visa estudar o sistema nervoso central, desvelando como ocorre o seu funcionamento, estrutura, desenvolvimento e as alterações ocorridas ao longo da vida do ser humano.

Os estudos realizados desde o século XVIII ao século XXI apontam que a neurociência se integra à outras ciências numa rede que amplia as informações e constrói um conhecimento que parece não se esgotar. O termo neurociência se difunde como um conceito transdisciplinar ao reunir diversas áreas de conhecimento no estudo do cérebro humano. As dificuldades decorrentes de tempos diversos de conhecimento, neurociência e educação, diluem-se na medida em que cada um se apropria das terminologias do outro e buscam um novo conhecimento. (Oliveira, 2011, p.20).

Diante disso, é importante esclarecer que o sistema nervoso é constituído a partir de três elementos: cérebro, coluna vertebral e os nervos periféricos, uma área pautada na psicologia e biologia. (Oliveira; 2014; Bartoskeck, 2007, Apud Cardoso; Queiroz, 2019). Para um melhor entendimento, a figura abaixo faz uma demonstração do Sistema Nervoso e suas dimensões central e periférica:

Figura 1: Sistema Nervoso.

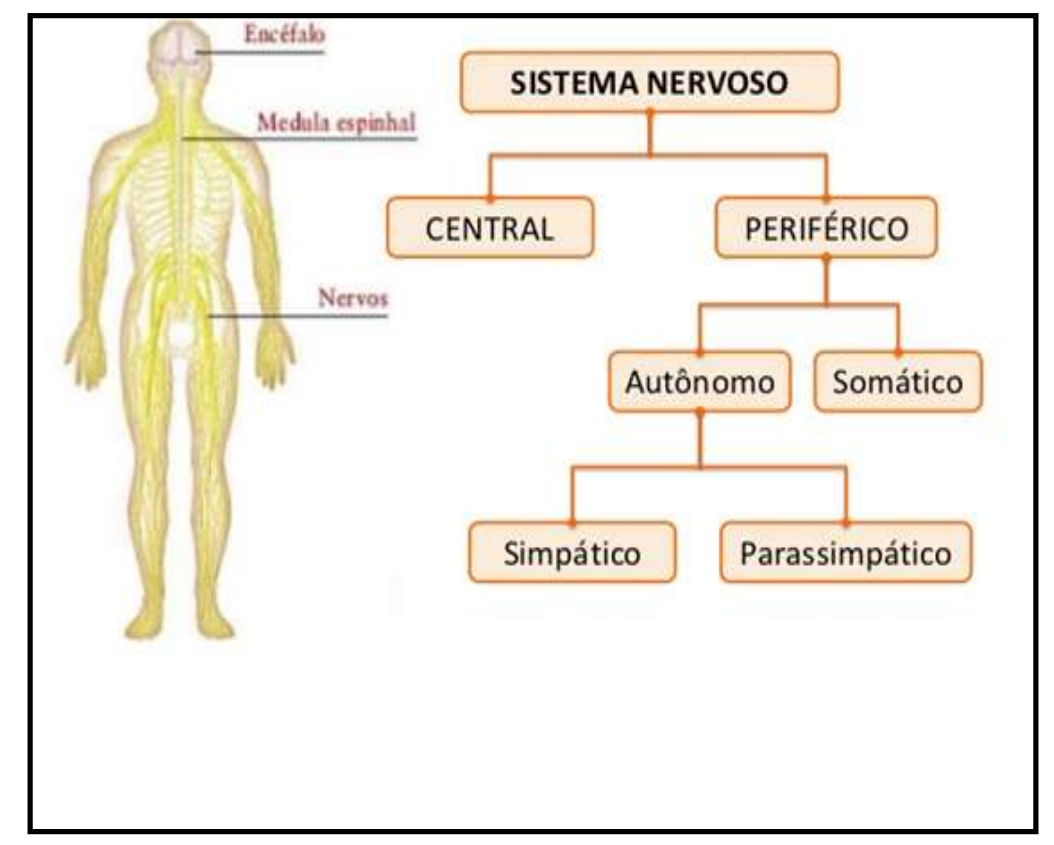

$<\mathrm{http} / / /$ wesleibio.blogspot.com/p/neuroanatomia.html>. Adaptado pelos autores (2021).

A Neurociência é conhecida como uma área que estuda o sistema nervoso central (SNC) e suas ações no corpo humano, estando articulada com várias áreas do conhecimento como, biomedicina, filosofia, bioquímica, farmacologia [...], considerandoa, portanto, como uma ciência multidisciplinar. Por isso, torna-se uma ciência de extraordinária complexidade para o seu entendimento, mas de grande relevância para o professor de ensino e aprendizagem. (Alves 2017; Cardoso; Queiroz, 2019). Essa ciência que visa uma melhor compreensão de como funciona o sistema nervoso central, surge como um elo entre teoria e prática docente, que tem contribuído para um melhor entendimento de como o aluno pode desenvolver sua aprendizagem.

Porém, destaca-se que a neurociência não tem a pretensão de criar teorias, mas contribuir com aqueles que buscam compreender como funciona os mecanismos cerebrais articulados a aprendizagem, e com isso, estimular para busca de metodologias que possam possibilitar novas alternativas para a prática docente. Sendo assim, educação e neurociência tornamse uma via de mão dupla. (Oliveira, 2011).

Dentre os campos de estudo da Neurociência, tais como: (1) Neuropsicologia, que se propõe estudar a interação entre as ações do nervo e as funções ligadas à área psíquica; (2) Neurociência comportamental, que pesquisa a ligação entre o contato 
do organismo e seus fatores internos (emoções e pensamentos) ao comportamento visível, como a forma de se falar e os gestos, entre outros, está ligada à psicologia comportamental; (3) Neuroanatomia, que estuda toda estrutura do sistema nervoso, fragmentando o cérebro, a coluna vertebral e os nervos periféricos externos, busca analisar cada item para e apreender a respectiva função de cada parte e nomeá-la, Neurofisiologia, que examina as funções ligadas as diferentes áreas do sistema nervoso e (4) Neurociência cognitiva, estuda a capacidade cognitiva do indivíduo como o raciocínio, abarcando também a memória e o aprendizado. (Marque, 2016 Apud Cardoso; Queiroz, 2019). (Grifo nosso).

Das áreas de conhecimento supracitadas, a neurociência cognitiva é foco desta pesquisa, pois é por meio do entendimento de sua funcionalidade que o professor poderá desenvolver um trabalho diferenciado no contexto escolar, junto aos seus alunos. Isso porque, para trabalhar com o discente em fase desenvolvimento, é preciso ter uma visão holísticas das mais variadas formas de aprendizagem. Dessa forma, o campo de investigação da neurociência se desenvolve rapidamente e tem se ampliado, interessando autores de diversas áreas, inclusive professores. (Oliveira, 2011).

[...] os conhecimentos em neurociência são produzidos numa velocidade vertiginosa e a possibilidade de comprovação científica de suas afirmações conta com importantes exames de Neuroimaggem com tecnologia de ponta. São conhecimentos que se renovam, transformam-se e criam novos paradigmas. A cada instante se esclarecem mais e mais o funcionamento desta fascinante estrutura que é o cérebro humano. (Oliveira, 2011, p.09).

Para o autor, a neurociência é um campo de investigação que visa estudar como o cérebro funciona, e com isso a educação passa a ser uma ciência do ensino e da aprendizagem, onde elas passam a interacionar-se, pois o cérebro tem uma importância significativa para a aprendizagem dos alunos. Dessa forma, para a Neurociência não se pode reduzir mente/cérebro a uma maquinaria cognitiva, com processamento de informações, tendo em vista que capacidade de expressão e emoção é peculiar ao gênero humano, diferente de um computador. É por meio dessa visão de trabalho que a neurociência cognitiva e educação toram-se campos do conhecimento importantes para impulsionar a aprendizagem do aluno.

\section{Neurociência e Educação}

A descoberta da Neurociência tem demonstrado campos desconhecidos do encéfalo e suas dimensões. E essa descoberta tem contribuído para o campo educacional, em especial para aprendizagem do aluno. Para Oliveira (2011), a neurociência como ciência interdisciplinar, por exemplo, entende que a educação é o eixo central do desenvolvimento humano, por ser vista como uma área que atua sobre o cérebro humano como ainda não vista em outras áreas do conhecimento, portanto:

[...] a Neurociência busca entender como o cérebro aprende e como o mesmo se comporta no processo de aprendizagem, são definidos métodos para identificar como os estímulos do aprendizado podem chegar neste órgão central. Sabe-se que os estados mentais são provenientes de padrões de atividades neural, então, a aprendizagem é alcançada por meio da estimulação das conexões neurais, que podem ser fortalecidas dependendo da qualidade da intervenção pedagógica. (Alves, 2017, p.321).

Por isso, é importante uma ação didática diferenciada por parte do professor, buscando compreender como essa ciência pode ajudar no processo de ensino e aprendizagem. O professor em sua prática docente precisa buscar alternativas diferenciadas, utilizando métodos de acordo com as especifidades de sua turma ou aluno, pois a aprendizagem é pessoal em cada sujeito singular, com suas particularidades. No campo educacional, a neurociência tem muito a contribuir com o trabalho do professor que por meio dessa nova alternativa de trabalho, terá nas mãos conhecimentos suficientes para instigar a aprendizagem do aluno. Nessa perspectiva, a aprendizagem resulta de alterações cerebrais decorrentes de experiências, que se impulsionadas de forma correta levará a uma aprendizagem significativa por parte dos discentes. 
Na educação, a neurociência passou a ser vista de forma diferenciada a partir dos estudos publicados pela Organização de Cooperação e Desenvolvimento Econômico OCDE, no ano de 2003, que apontou uma visão inovadora para a aprendizagem com base em pesquisas sobre o cérebro. Mas, depois de 10 anos de estudos, conhecido como a década do cérebro, descobriu-se que esse tempo não foi suficiente para a exploração do cérebro humano, entendendo-se que havia mais campos desconhecidos que precisariam ser estudados. Nesse sentido, é perceptível que na conjuntura atual, mesmo com toda as técnicas utilizadas para aprimorar o entendimento acerca da funcionalidade do cérebro, existe mistérios que precisam ser descobertos. (Bortoli; Terya, 2017).

Além disso, Relva (2010), acrescenta que a neurociência é compreendida como neuroeducação e a aprendizagem passa a adquirir novas perspectivas por conta dos avanços tecnológicos. As técnicas utilizadas para o estudo do cérebro humano permitem conceber as regiões ativas no momento de sua execução, permitindo um melhor aprofundamento de conhecimentos sobre o cérebro que, na anterioridade, eram apenas dedutivas por meio de observações externas. (Apud Bortoli; Terya, 2017).

Diante disso, observa-se que a neurociência constituída posteriormente como neuroeducação por meio de técnicas voltadas para o desenvolvimento da aprendizagem, tem somado para um melhor entendimento de como o aluno aprende. "O cérebro humano caracteriza-se como o principal órgão do encéfalo, localizado no sistema nervoso central, desempenhando o controle de atividade voluntárias e involuntárias em razão dos estímulos provenientes do meio ambiente (Relvas, 2009; Maia, 2011 Apud Bortoli; Terya, 2017, p.73). (Grifo nosso).

O meio ambiente é fundamental para aprendizagem do aluno, pois por meio da interação social e contato direto com o objeto, o discente conhecerá a regra do jogo e com isso, desenvolverá sua aprendizagem. Contudo, é relevante salientar que essa aprendizagem só será efetiva se houver estímulos por parte do professor, do meio em que o aluno está inserido. A aprendizagem para ser significativa, precisa fazer sentido para o aluno, e é nesse sentido que a neurociência pode contribuir, principalmente com o uso da técnica mnemônica 5 .

Tomando como base todos os conceitos discutidos até então, faria sentido que as escolas, individualmente, iniciassem um processo de alteração de suas políticas educacionais e promovessem mudanças curriculares e na formação de seus professores. De fato, um número crescente de escolas tem procurado inovar seus métodos de ensino, afim de conciliar não apenas a formação de indivíduos inovadores, mas também aliar as ferramentas tecnológicas disponíveis (e que são altamente atrativas) ao ensino. Muitas dessas inovações passam pela promissora ideia de buscar métodos educacionais que levem em consideração os mecanismos neurais de aprendizagem [...] (Carvalho; Boas, 2011, p.236).

As escolas enquanto instituições sociais têm um papel fundamental no processo de ensino e aprendizagem. Porém, para que realizem um trabalho com vista a atingir objetivos positivos e metas traçadas no início do ano, é preciso inovar em suas práxis educativas, criando alternativas de trabalho que sejam diferenciadas daquelas trabalhadas de forma tradicional no cotidiano escolar. É preciso, acima de tudo, criar estratégias de trabalho que possa estabelecer uma relação de intimidade, entre professor e aluno, entre ensino e aprendizagem, para que o conhecimento seja de fato construído. É nessa relação que a neurociência vem conquistando espaço no campo educacional, pois:

[...] fascinam cada vez mais pessoas pela possibilidade de compreensão dos mecanismos das emoções, pensamentos e ações, doenças e loucuras, aprendizado e esquecimento, sonhos e imaginação, fenômenos que nos definem e constituem. Mas concretamente, os profissionais de saúde, terapeutas, professores e legisladores podem agora se apropriar da imensa massa de dados empíricos sobre genes, proteínas, células, circuitos e organismos inteiros. (Ribeiro, 2013, p.7). (Grifo nosso).

\footnotetext{
${ }^{5}$ É um auxiliar de memória. São tipicamente, verbais, e utilizados para memorizar listas ou fórmulas, e baseiam-se em formas simples de memorizar maiores construções, baseados no princípio de que a mente humana tem mais facilidade de memorizar dados quando estes são associados a informação pessoal, espacial ou de caráter relativamente importante, do que dados organizados de forma não sugestiva) para o indivíduo) ou sem significado aparente. Porém, estas sequências têm que fazer algum sentido, ou serão igualmente difíceis de memorizar. < https://pt.wikipedia.org/wiki/Mnem\%C3\%B3nica>. Acessado em: 15/09/20.
} 
A neurociência abre um leque de possibilidades para as diversas áreas do conhecimento, e no âmbito educacional lança alternativas para o professor realizar o trabalho diferenciado, "alimentando o debate sobre a relação entre neurociências e educação, tais como as evidências de que o direcionamento da atenção do aluno para os pontos específicos do material estudado favorece a retenção de memórias" [...] (Ribeiro, 2013, p.10). Para a neurociências os gestos não verbais antecipam os saltos cognitivos, destacando que o aprendizado linguístico com grafemas e morfemas tem resultados bem mais positivos com relação ao ensino de palavras, permitindo pensar estratégias pedagógicas, tendo como pressuposto o estudo do cérebro humano.

\section{Memória, Aprendizagem e Motivação}

Estudos no campo da neurociência tem demonstrado que a motivação contribui para a aprendizagem, isso porque auxiliam a passagem de curta para uma memória de longo prazo. [...] "A motivação é responsável pela liberação de sustâncias responsável por mobilizar a atenção e reforçá-la na relação com o objeto que a afetou”. (Brotoli; Terya, 2017, p.70). Porém, ressalta-se que ainda há caminhos a serem percorridos, com necessidades de novas pesquisas, que faça indicativos de uma maior aproximação entre neurociência e educação, que possam diluir mitos sobre essas duas áreas do conhecimento.

A memória faz de nós aquilo que somos e podemos vir a ser, pois cada lembrança recordada ou esquecida faz com que sejamos sujeitos únicos, uma vez que, para duas pessoas, vivenciando a mesma situação, a forma como esse momento será armazenado será distinta, levando a pontos de vista diferentes que, por sua vez, trarão recordações diferentes. O conjunto de memórias de cada pessoa influencia a sua personalidade. [...]. (Sousa; Salgado, 2015, p.142).

A memória é um campo do conhecimento com enigmas a serem desvendados. Ela tem a capacidade de gerar aprendizado ou não da informação, dependendo das conexões entre memória com os sistemas mnemônicos. Para o autor, esses sistemas são técnicas utilizadas no processo de memorização, podendo ser também mecanismos criado pelo próprio cérebro, que auxiliam no armazenamento, resgatando posteriormente as informações. A memória é de grande relevância para a vida do ser humano e sua aprendizagem, sendo esses elementos parte do sistema nervoso cerebral.

Nosso cérebro recebe constantes estímulos externos através do nosso sistema sensorial que consiste em: tato, olfato, visão audição e paladar. O cérebro capta essas informações que chegam a todo o momento, as processam, selecionam e estabelece quais informações serão armazenadas ou deletadas da memória. A memória é um elemento de extrema importância no processo de aprendizagem, estando, portanto entrelaçadas. (Cardoso; Queiroz, 2019, p.37).

Os estímulos afetam diretamente as atividades nervosas, fazendo com que aprendemos a desenvolver desde os movimentos simples ao mais complexo, tais como, caminhar, amar e pensar, precisando, portanto, executá-las. Sem a função da memória, o ser humano teria dificuldade para aprender, tendo que reaprender constantemente, as atividades triviais do cotidiano. (Cardoso; Queiroz, 2019). Quanto mais a memória se consolida, será evidente que haverá mais aprendizagem, uma ação inerente a vida humana. Porém, para que essa mesma aprendizagem possa de fato ocorrer, o ambiente assume um papel fundamental nesse processo. Um ambiente carregado de estímulos, facilita a apreensão da aprendizagem do aluno em sala de aula.

Quando passamos a tratar de motivação em sala aula, um elemento de grande poder persuasivo é a pessoa do professor. No processo motivacional é importante considerar a relação entre professor e aluno junto ao clima estabelecido pelo professor, da relação empática com seus alunos, de sua capacidade de ouvir, refletir, discutir o nível de compreensão dos mesmos e da criação das pontes entre o seu conhecimento e o deles. Sendo assim, proporcionará a participação dos alunos nas aulas, o que é de suma importância, pois trarão oportunidades de expressar seus conhecimentos, preocupações, interesses, desejos e vivências, participando de forma ativa e crítica na construção e reconstrução de sua cultura. (Gómez, 2000 Apud Padilha; Carvalho, 2017, p.31). 
O professor é um profissional que em sua prática cotidiana tem a responsabilidade pelo processo de ensino e aprendizagem dos alunos em sala de aula, recaindo sobre ele, a tarefa de motivar os alunos para busca do conhecimento. A negativa desse trabalho, pode afetar o desempenho não só do aluno, mas de toda uma turma. Por isso, ser professor na atualidade requer conhecer e compreender como se processa a neurociência, para contribuir com a formação de um aluno partícipe do processo de sua aprendizagem.

Deci e Rayan (2000), canalizam para o mesmo entendimento, mas sublinham que motivação é uma característica de cada professor, construída ao longo da prática docente. Todavia, mesmo com toda uma bagagem declinada para esse entendimento, uma sala com inúmeros alunos, tempo de profissão, concepções ideológicas e os fatores contextuais, podem afetar todo o desempenho do professor como de sua turma. "[...] a interação dos professores com seus alunos vai além das disposições pessoais, uma vez que envolve vários outros fatores como por exemplo a sua percepção acerca do envolvimento dos estudantes. (Apud Padilha; Carvalho, 2017, p.3131).

A motivação depende também de fatores pessoais, relacionado ao querer do aluno, suas atitudes, crenças e experiências, provenientes de fatores externos. De acordo com a neurociência cognitiva, que tem como foco de trabalho a compreensão cerebrais e dos processos de cognição, o processo de aprendizagem não resulta apenas de armazenamentos de elementos perceptuais, mas sim, do processamento e preparação das informações oriundas das percepções do cérebro. (Carvalho, 2011).

O indivíduo, vive permanentemente em busca de respostas para as suas percepções, pensamento e ações, tem suas conexões neurais em constante reorganização e seus padrões conectivos alterados a todo momento, mediante processos de fortalecimento ou enfraquecimento de sinapses. No cérebro, há neurônios prontos para a estimulação. A atividade mental a reconstrução de conjuntos neurais, processando experiências vivenciais e/ou linguísticas, num fluxo e refluxo de informação. As informações, captadas pelos sentidos e transformadas em estímulos elétricos que percorrem os neurônios, são catalogadas e arquivadas na memória. É essa capacidade de agregar dados novos a informações já armazenadas na memória, estabelecendo relações entre o novo e o já conhecido e reconstruindo aquilo que já foi aprendido, num processamento constante das interpretações advindas da percepção, que caracteriza a plasticidade do cérebro (Izquierdo, 2002; Lent, 2001; Ratey, 2001 Apud Carvalho, 2011, p.540).

Assim, o cérebro tem uma dinamicidade que por meio dos neurônios que estão prontos para estimulação, caracterizam a unicidade do desenvolvimento humano, podendo ser visto a partir de sua complexidade, vivendo em constante busca por respostas para as suas percepções, requerendo dessa forma, "competências para lidar de forma organizada com as informações novas, ou com aquelas já armazenadas no cérebro, a fim de realizar novas ações”. (Carvalho, 2011,p.540).

Aprender, exige, portanto, o cumprimento de planos pré-estabelecidos, resultados de ações mentais desenvolvidas, ensinadas mentalmente e que entusiasmam o planejamento de atitudes futuras. Sendo assim, o "cérebro está organizado para funcionar com o feedback interno e externo, sendo autorreferente, isto é, o que é recebido em qualquer nível cerebral dependerá de tudo o que advier nesse nível, e o que é informado para o nível seguinte vai depender do que já estiver ocorrendo nesse nível" (Ratey, 2001 Apud Carvalho, 2011).

\section{Formação de Professore a partir da Neurociência}

O professor como agente de transformação social, precisa estar antenado às mudanças ocorridas na sociedade, acompanhando as inovações e pesquisas para o campo educacional, buscando na ação didática, qualificar-se para atender o aluno da atualidade. Para a formação de professores, é preciso um domínio técnico para a busca de soluções no que tange aos problemas vivenciados no dia-a-dia da escola. Em seus estudos, Oliveria (2011) descreve que o cérebro é moldável pelos estímulos, decorrente do próprio organismo, da programação genética e do ambiente externo. Diante disso, tornar-se imprescindível, para a formação do professor, investimento em conhecimentos que o habilitem para ensinar, motivar e avaliar o aluno em um formato 
mais eficiente. Desse modo, dos saberes docentes, a neurociência serve de base para o ensino, como competência de seu "saberensinar".

Se os saberes dos professores possuem certa coerência, não se trata de uma coerência teórica nem conceitual, mas pragmática e biográfica: assim como as diferentes ferramentas de um artesão, eles fazem parte da mesma caixa de ferramentas, pois o artesão que os adotou ou adaptou pode precisar deles em seu trabalho (Tardif, 2002, p.64 Apud, Oliveira, 2011, p.19).

O professor com seus ensinamentos e prática cotidiana, além da missão de ensinar, por meio de motivação, pode fazer um diferencial no contexto de trabalho, fortalecendo o vínculo com seus aprendizes e com isso, promover êxitos no processo de ensino e aprendizagem dos discentes. Porém, o que tem se observado é que, "uma das maiores inquietações do professor contemporâneo é de como fazer com que o aluno aprenda de forma efetiva". (Cardoso; Queiroz, 2019, p.43). É por esse caminho que a neurociência traz consideráveis contribuições para a prática docente, haja vista que enxerga o aluno pelo viés da singularidade, único e capaz de colaborar com o entendimento da estrutura do cérebro.

\section{As Contribuições da Neurociência para a Aprendizagem}

A neurociência com sua metodologia de trabalho contribui significativamente para a aprendizagem o aluno, oportunizando ao professor conhecimentos suficientes para a elaboração de estratégias para melhorar a sua prática educativa. Essas informações podem influenciar aspectos de natureza social, psicológicos e culturais, a partir do estudo realizado pelo professor. Mas para que haja uma contribuição eficaz, é importante levar em consideração o ambiente em que o aluno está inserido, com infraestrutura adequada, materiais didáticos que correspondam aos anseios dos alunos e disposição por parte professor para desenvolver um trabalho com novos olhares em prol de uma educação diferenciada e de qualidade.

O processo de aprendizagem é imprescindível em qualquer etapa na vida do ser humano, bem como, vem se desenvolvendo desse os primórdios de sua vida. A neurociência tem desmontado o quão promissor pode ser uma parceria com a educação, trazendo todo o seu conjunto de saberes sobre o Sistema nervoso Central, local onde tudo acontece, desde os comportamentos, pensamentos, emoções e movimentos, e é a partir dos conhecimentos desta área que a educação pode ter um salto quando se fala em efetividade e eficácia, levando em conspiração que a partir do surgimento e avanço da neurociência foi possível fornecer melhorias na qualidade de vida da sociedade atual, disponibilizando tratamento efetivos para variados distúrbios neurológicos, ou seja, contribui e tem contribuído significativamente para o desenvolvimento de soluções de diversos transtornos e doenças, incluindo os problemas educacionais. (Santos; Sousa, s/d, p.2).

É por meio desse viés que a neurociência aliada à educação pode transforma-se em um potencial de relevância imprescindível, capaz de desmontar o trabalho tradicional realizado nas instituições de ensino, e por meio de estratégias inovadoras com vista a realizar um trabalho conectado a biologia cerebral, o discente terá nas mãos a oportunidade de desenvolver suas potencialidades e com isso, aprender de forma tranquila e harmoniosa, sem imposições, mas por estímulos.

Diante disso, é preciso a criação de estratégias que promovam em sala de aula atividades que desenvolvam o sistema nervosos central, por meio de estímulos externos, com a intenção de promover as sinapses com a utilização de jogos e de músicas em sala de aula, que excitem várias funções mentais, salientando que atividades prazerosas, lúdicas e desafiadoras também fortalecem as sinapses. (Santos; Sousa, s/d).

Para alunos de primeiro e segundo segmento é interessante que o professor utilize como recursos jogos que estimules o raciocínio lógico, que envolvam a concentração, a atenção, a motivação. O xadrez, o jogo de damas, o dominó, são exemplos de possibilidades didáticas, assim como enigmas, trilhas, jogos verbais, entre outros. O contato com a arte é essencial, pois essas atividades provocam mudanças na estrutura cerebral diante de uma apreciação e releitura de obras 
clássicas e contemporâneas. Vivências fílmicas, musicais e teatrais são elementos importantes na linguagem e na ampliação de estímulos neurais. (Cardoso; Queiroz, 2019, p.44).

É notório que há dificuldades para o desenvolvimento da aprendizagem do discente da atualidade, com vivências divergentes de outrora, com conhecimentos tecnológicos muitas vezes superior ao do professor. Mas se o professor tiver conhecimento da neurociência, mais especificamente da neuroeducação, poderá desenvolver um trabalho diferenciado daqueles comumente realizado na escola do século XIX. É preciso um olhar diferenciado com as atividades propostas aos alunos, é preciso ter consciência e conhecimento de recurso que podem proporcionar um maior alargamento da aprendizagem cerebral dos alunos. Os jogos, as artes, com suas similitudes são ferramentas necessárias para o despertar da aprendizagem do aluno.

\section{Considerações Finais}

A pesquisa sobre as contribuições da neurociência para a prática docente demonstrou o quanto essa área do conhecimento pode contribuir para o desenvolvimento do trabalho do professor no ambiente escolar. Porém, é importante deixar claro nessa escrita nada conclusivo, mas um indicativo de que a neurociência e a educação podem fortalecer o trabalho do professor por meio de estratégias de trabalhos que estimulem a aprendizagem do aluno.

Apesar de o cérebro humano ser um campo que precisa de um estudo mais aprofundado, compreende-se que o conhecimento sobre ele até aqui abordado tem sido relevante para o desenvolvimento de um trabalho diferenciado e de uma aprendizagem significativa. Portanto, é preciso ressaltar que ainda é perceptível a dificuldade por parte do professor para compreensão dessa nova área de conhecimento, mas que se compreendida como preceituada nas análises textuais, o trabalho poderá canalizar para um novo entendimento e com isso, proporcionar uma aprendizagem harmoniosa e eficiente juntos aos alunos no âmbito escolar.

Dessa forma, os resultados da pesquisa contribuirão com a coletânea já existente e com aqueles profissionais e pesquisadores que buscam aprofundar o entendimento sobre essa área do conhecimento que a cada dia vem impulsionando um olhar diferenciado acerca do processo de ensino e aprendizagem os discentes no ambiente escolar, pois essas informações são relevantes para que o professor possa melhorar sua prática docente e com isso, compreender como se processa a aprendizagem do aluno em suas mais distintas abordagens.

\section{Referências}

Alves, R. R. N., Sousa, A. M., \& Oliveira, P. de. (2017). A Neurociência na formação dos educadores e sua contribuição no processo de aprendizagem, <http://pepsic.bvsalud.org/pdf/psicoped/v34n105/09.pdf>.

Bortoli, B. de, \& Terya, T. K. (2017). Neurociência e educação: os percalços e possibilidades de um caminho em construção, <file:///D:/Users/CLIENTE/Downloads/32171-Texto\%20do\%20artigo-156632-1-10-20170119.pdf>.

Cardoso, M. A., \& Queiroz, S. L. (2019). As contribuições da Neurociência para a Educação e a formação de professores: Um diálogo necessário. Cadernos da pedagogia, v. 12, <file:///D:/Users/CLIENTE/Downloads/1238-3588-2-PB.pdf>.

Carvalho, D. de, \& Boas, C. A. V. (2018). Neurociências e formação de professores: reflexões na educação e economia, <https://www.scielo.br/pdf/ensaio/v26n98/1809-4465-ensaio-26-98-0231.pdf>.

Carvalho, F. A. H. de. (2021). Neurociências e Educação: Uma articulação necessária na formação docente. Trab. Educ. Saúde, 8. <https://www.scielo.br/pdf/tes/v8n3/12.pdf>.

Gil, A. C. (1946). (2002). Como elaborar projetos de pesquisa. (4a ed.). Atlas.

Godoy, A. S. (1995). Pesquisa qualitativa, tipos fundamentais. Revista de Administração de Empresas. v. 35.

Oliveira, G. G. de. (2011). Neurociências e os processos educativos: Um saber necessário na formação de professores, <https://www.uniube.br/biblioteca/novo/base/teses/BU000205300.pdf>.

Padilha, C. de M., \& Carvalho, A. H. de. (2017). A motivação na aprendizagem à luz da teoria de FOGG: Contribuições das Neurociências, <https://sistemas.furg.br/sistemas/sab/arquivos/bdtd/0000011972.pdf>. 
Research, Society and Development, v. 10, n. 12, e392101218775, 2021

(CC BY 4.0) | ISSN 2525-3409 | DOI: http://dx.doi.org/10.33448/rsd-v10i12.18775

Ribeiro, S. Neurociências: tempo de cérebro. Estudos avançados (2013). <https://www.scielo.br/pdf/ea/v27n77/v27n77a02.pdf>.

Santos, C. P. dos, \& Sousa, K. Q. A neuroedução e suas contribuições às práticas pedagógicas contemporâneas. <https://eventos.set.edu.br/e nfope/article/viewFile/1877/777>.

Sousa, A. B. de, \& Salgado, T. D. M. (2015). Memória, aprendizagem, emoções e inteligência. <https://www.lume.ufrgs.br/bitstream/handle/10183/1 32515/000982720.pdf? sequence=1>. 